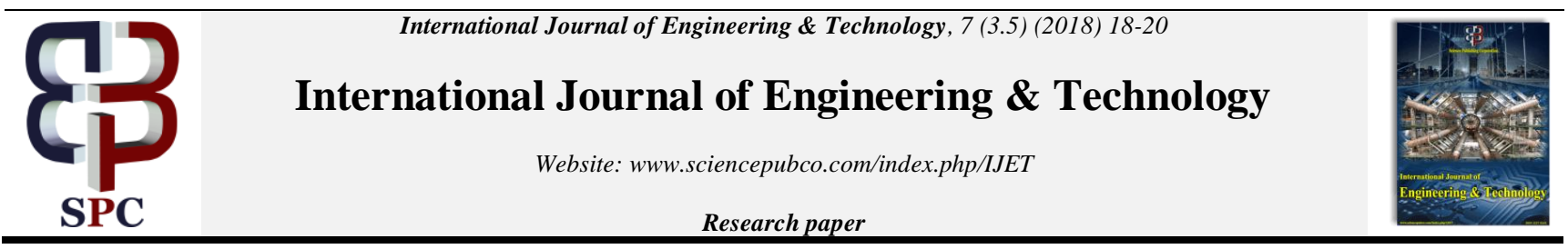

\title{
Clear and Fuzzy Neighborhood Models of Wastewater Treatment
}

\author{
A.M. Smyrin ${ }^{1 *}$, I.A. Sedukh ${ }^{2}$ \\ Lipetsk state technical University, Russia \\ 1,2 Orcid: 0000-0001-8454-6032, 0000-0003-0012-8103
}

\begin{abstract}
The paper describes the definition of wastewater and their main types, shows the main components of the sewage treatment plant. The process of wastewater treatment is presented using clear and fuzzy dynamic neighborhood models, their comparative characteris tics are given, and a conclusion is drawn.
\end{abstract}

Keywords:

\section{Introduction}

Surrounding models [1-11] generalize such traditional discrete models, such as finite automata, cellular automata, Petri nets and others, and allow to model complex distributed processes. In this case, the structure of the process is divided into a finite set of interconnected nodes, for each of which are defined neighborhoods by inputs, states and outputs.

Let's consider an example of the use of clear and fuzzy dynamic occlusal models for representing the wastewater treatment process.

\section{Wastewater Treatment System}

Sewage - a collection of any water and precipitation used in the home and on the territory of industrial enterprises, whose properties were unclaimed or deteriorated as a result of human activities, resulting in water being diverted to water bodies [12].

The composition of industrial and household waste water pollution includes mineral, organic impurities and their mixtures, biological impurities. Mineral in its composition contains components of sand, clay and other fossil rocks. The main constituents of organic impurities are vegetable and animal substances, oil products, pesticides, heavy metals, phenols, etc. Biological impurities include microorganisms, plants, yeasts, molds, etc. The concentration of pollutants depends on the nature of the production, type Produced products, especially the technological process of production.

For sewage treatment, treatment facilities are used, representing a chain of interconnected objects: lattices with a gap between the 10 $\mathrm{mm}$ rods for separating large debris; Aerated sand grains for sand removal; primary sedimentation tanks for sedimentation and removal of floating substances (fats, oil products); reservoiraverager for the averaging of effluents by the quantitative flow and indications of LV; Two bioreactors for biological purification with nitriding-denitrification processes; An intermediate reservoir for the accumulation of conditionally pure water before purification on the post-treatment filters and installation of ultra-violet irradiation; Reservoir of clean water before discharge into a water body.

By a gravity collector, wastewater is fed into the lattice building, in which mechanical filtration is carried out on lattices with a 10 $\mathrm{mm}$ cell from large debris. On the open channel after the building of the grates, the sewage falls on the aerated sand trap. The sand settling in the lower part is pumped by submersible pumps to the pressurization facilities in the sand bunker building. Sewage through open channels enters the primary stands.

In the primary sedimentation tanks, final purification from suspended particles and the primary stage of anaerobic digestion take place, after which waste water enters the reservoir-averager. In the averager, continuous mixing of heterogeneous effluents is carried out with the help of two special immersion mixers, which ensure optimum mixing of wastewater with the dosing reagents. Control of the supply of reagents is carried out according to the $\mathrm{pH}$ sensor. With the pumping group, waste water is fed to biological reactors of variable action such as SBR. In biological reactors there is a major process of removing contaminants from sewage. The aerobic sludge is pumped out to the sludge collector with pumps. Conditionally pure water is supplied to the storage tank. From the storage tank water is supplied to the final cleaning in the combined sand filters and ultraviolet disinfection. Purified sewage flows into the reservoir of pure water by gravity and then into the river.

We model the sewage treatment process using clear and odd dynamic neighborhood systems.

\section{Clear and Fuzzy Neighborhood Models of Wastewater Treatment}

In [7] a generalized definition of the neighborhood model was introduced.

Further development of the theory of neighborhood systems is fuzzy-neighborhood models [4], which take into account the degree of fuzzy influence of each other elements of neighborhoods. 
The fuzzy dynamic neighborhood model in the general case is described by a set:

$N S_{G}(\omega)=(N(\omega), X(\omega), V(\omega), Y(\omega), Z(\omega), G(\omega), F(\omega), X(\omega)[0], \tau(\omega))$ ( $\omega_{-}$an indication of fuzziness), where [6]: 1) $N(\omega)_{\text {- fuzzy }}$ structure; 2) $X(\omega)$ - vector of fuzzy states defined by the membership functions; 3) $V(\omega)_{\text {- vector of fuzzy controls; 4) }} Y(\omega)$ the vector of fuzzy outputs; 5) $Z(\omega)$ - a vector of fuzzy time delays at the nodes; 6) $G(\omega): X_{O_{x}(\omega)}(\omega) \times V_{O_{v}(\omega)}(\omega) \rightarrow X(\omega)$ fuzzy function of the conversion conditions neighborhood models, $X_{O_{x}(\omega)}(\omega)$ and $V_{O_{v}(\omega)}(\omega)$ - a fuzzy sets of states and controls of nodes entering the neighborhoods $O_{X}(\omega)$ and $O_{V}(\omega)$ accordingly; 7) $F(\omega): X_{O_{x}(\omega)}(\omega) \times V_{O_{v}(\omega)}(\omega) \rightarrow Y(\omega)$ _ fuzzy function of recounting the outputs of the model; 8) $X(\omega)[0]$ initial state of the model; 9) $\tau(\omega)_{\text {_ fuzzy time of the system }}$ functioning.

Select five nodes of the neighborhood model $A=\left\{a_{1}, \ldots, a_{5}\right\}$, where ${ }^{a_{1}}$ - the node of the input data, $a_{2}$ - the primary settler unit, ${ }^{a_{3}}$ - the aggregator node, ${ }^{a_{4}}$ - node bioreactor №1, ${ }^{a_{5}}$ node bioreactor №2.

In Fig. 1 shows the dependence of the states and outputs of the dynamic neighborhood model in each node at the next time point from states and inputs at the current time, where $v_{i j}=x_{i-1, j}$; $i=2, \ldots, 5, j=1, \ldots, 7$.

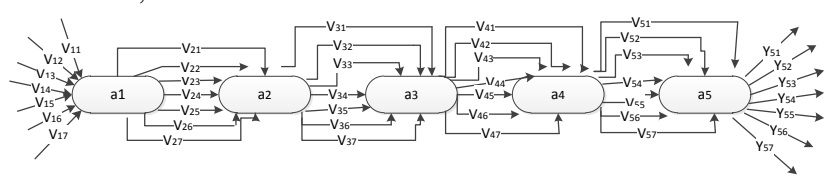

Fig. 1. Graph of the dynamic neighborhood model of the wastewater treatment process

Parametric identification of 4 models is carried out:

1) linear clear:

$$
\left\{\begin{array}{l}
X_{i k}[t+1]=\sum_{j=1}^{7} w_{i k j}^{x V} \cdot v_{i j}[t]+w_{i k}^{X} \\
Y_{5 k}[t]=\sum_{j=1}^{7} w_{5 k j}^{y V} \cdot v_{i j}[t]+w_{5 k}^{y}
\end{array}\right.
$$

where $i=1, \ldots, 4$-node numbers; $k=1, \ldots, 7, w_{i k j}^{x v}, w_{i k}^{x}, w_{5 k j}^{y v}$, $w_{5 k}^{y}$ - the parameters of the model;

2) fuzzy linear:

$$
\left\{\begin{array}{l}
X_{i k}[t+1]=\sum_{j=1}^{7} w_{i k j}^{x v}(\omega) \cdot v_{i j}[t]+w_{i k}^{x}(\omega) \\
Y_{5 k}[t]=\sum_{j=1}^{7} w_{5 k j}^{y v}(\omega) \cdot v_{i j}[t]+w_{5 k}^{y}(\omega)
\end{array}\right.
$$

where ${ }^{x k j}(\omega), w_{i k}^{x}(\omega), w_{5 k j}^{y v}(\omega), w_{5 k}^{y}(\omega)$ - the model parameters are defined the corresponding membership functions; 3) clear quadratic:

$$
\left\{\begin{array}{l}
X_{i k}[t+1]=\sum_{j=1}^{7} w_{i k j}^{X V} v_{i j}[t]+\sum_{j=1}^{7} \sum_{d=1}^{7} w_{i k j d}^{X W v} \cdot v_{i j}[t] v_{i d}[t]+w_{i k}^{X} \\
Y_{5 k}[t]=\sum_{j=1}^{7} w_{5 k j}^{y v} v_{i j}[t]+\sum_{j=1}^{7} \sum_{d=1}^{7} w_{5 k j d}^{y w v} \cdot v_{5 j}[t] v_{5 d}[t]+W_{5 k}^{y}
\end{array}\right.
$$

where ${ }^{W_{i k j d}^{x v v}}, W_{5 k j d}^{y v v}$ - the parameters of the model;

4) quadratic fuzzy:

$\left\{\begin{array}{l}X_{i k}[t+1]=\sum_{j=1}^{7} w_{i k j}^{x v}(\omega) \cdot v_{i j}[t]+\sum_{j=1}^{7} \sum_{d=1}^{7} w_{i k j d}^{x v V}(\omega) \cdot v_{i j}[t] \cdot v_{i d}[t]+w_{i k}^{x}(\omega) ; \\ Y_{5 k}[t]=\sum_{j=1}^{7} w_{5 k j}^{y v}(\omega) \cdot v_{i j}[t]+\sum_{j=1}^{7} \sum_{d=1}^{7} w_{5 k j d}^{y v v}(\omega) \cdot v_{5 j}[t] \cdot v_{5 d}[t]+w_{5 k}^{y}(\omega) .\end{array}\right.$

The criterion of the parametrical identification has the form:

$E=\frac{1}{35 N} \sum_{k=1}^{7}\left(\left\|Y_{5 k}[t] \quad \hat{Y}_{5 k}[t]\right\|^{2}+\sum_{i=1}^{4}\left\|X_{i k}[t+1] \quad \hat{X}_{i k}[t+1]\right\|^{2}\right) \rightarrow \min$ where $\hat{X}_{i k}, \hat{Y}_{5 k}-$ model values of states and outputs, respectively; $N_{-}$is the sample size. Note that the function $E$ is the mean square error of identification.

We present some results of modeling. The plots of the initial data and the results obtained from the models (1) - (4) for the outputs $Y_{51}[t]$ and $Y_{52}[t]$ are shown in Fig. 2-5.

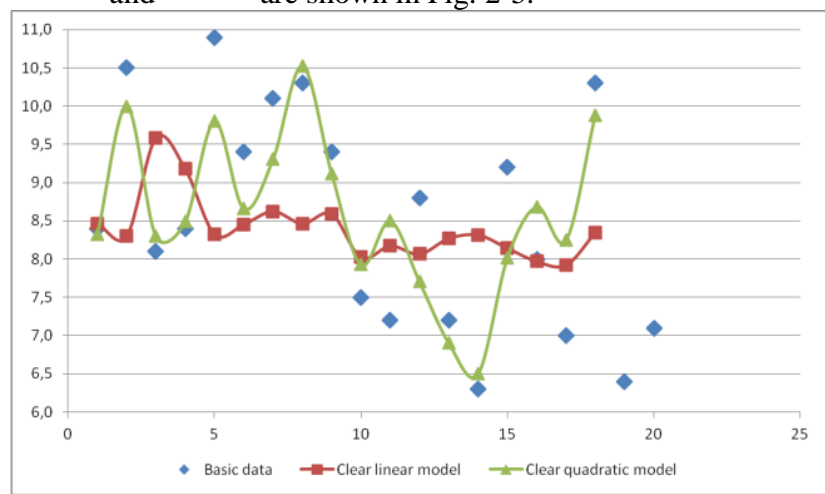

Fig. 2. The results of calculations on clear models for $Y_{51}[t]$

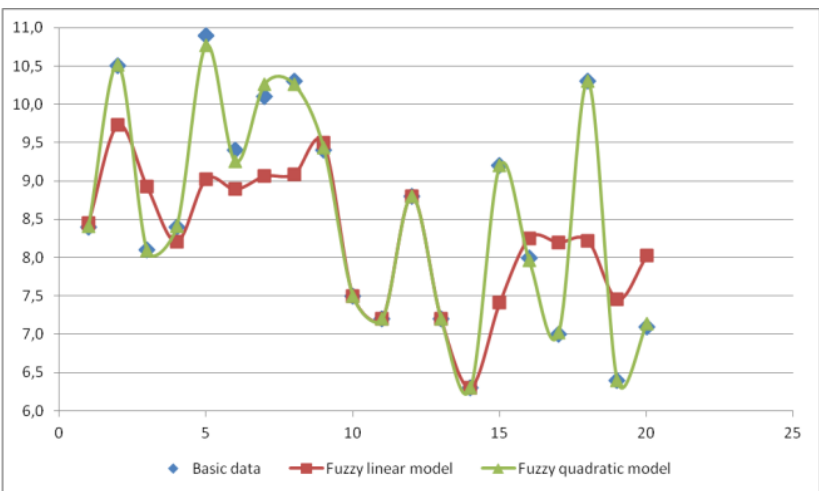

Fig. 3. The results of calculations on fuzzy models to $Y_{51}[t]$

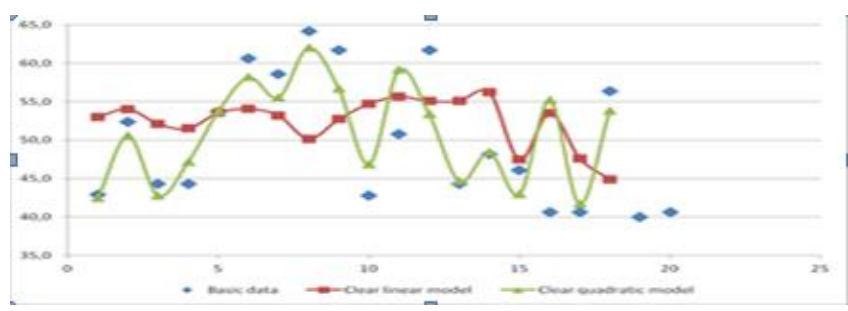

Fig. 4. The results of calculations on clear models for $Y_{52}[t]$ 


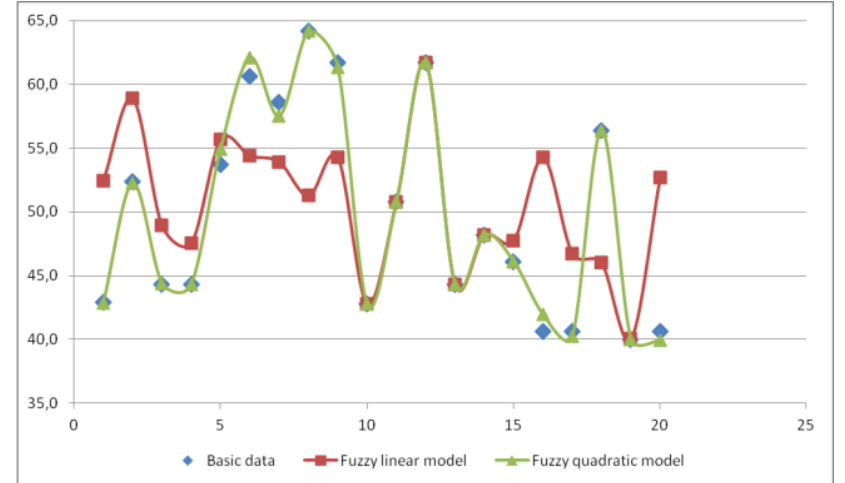

Fig. 5. The results of calculations on fuzzy models to $Y_{52}[t]$

A comparative characteristic of the mean square error of identification for the models considered is given in Table 1.

Table 1. Comparative characteristics of models

\begin{tabular}{|l|l|l|}
\hline \multicolumn{3}{|c|}{ Table 1. Comparative characteristics of models } \\
\hline Model & Clear & Fuzzy \\
\hline Linear & 24,1 & 5,5 \\
\hline Quadratic & 12,0 & 3,9 \\
\hline
\end{tabular}

From Table 1 it can be seen that the quadratic, clear and fuzzy dynamic neighborhood models provide the best result in comparison with the corresponding linear models in the sense of the mean square errors of identification. In addition, the introduction of fuzziness significantly improves the adequacy of clear neighborhood models.

\section{Conclusion}

A parametric identification of four dynamic neighborhood models of wastewater treatment was carried out. Their comparative characteristics in the sense of mean square errors are given. It is shown that the error of fuzzy neighborhood models is several times less than that of clear ones.

Acknowledgments: The work is supported by the Russian Fund for Basic Research (project 16-07-00854 a).

\section{References}

[1] Shmyrin, A.M., Sedykh, I.A, (2009), Identification and control algorithms of functioning for neighborhood systems based on petri nets, Automation and Remote Control, V. 71, No. 6, pp. 1265-1274

[2] Shmyrin, A.M., Sedykh, I.A, (2009), published in Upravlenie Bol'shimi Sistemami, No. 24, p. 18-33.

[3] Shmyrin, A.M., Sedykh, I.A, (2016), Neural Networks Neighborhood Models, Global Journal of Pure and Applied Mathematics, Vol. 12, No. 6, p. 5039-5046.

[4] Blyumin, S.L., Shmyrin, A.M, (2005), Neighborhood system. Lipetsk: LEGI, p. 132

[5] Blyumin, S.L., Shmyrin, A.M., Shmyrina, O.A, (2010), Bilinear neighborhood system. Lipetsk: LEGI, p. 130

[6] Blyumin, S.L., Shmyrin, A.M., Sedykh, I.A., Filonenko, V.Y, (2010), Neighborhood simulation of Petri nets. Lipetsk: LEGI, p. 124

[7] Shmyrin, A.M., Sedykh, I.A, (2012), Classification neighborhood bilinear models, Vestnik Tambov University. A series of Natural and technical Sciences. Tambov, T. 17. Vol. 5, pp. 1366-1369.

[8] Shmyrin, A.M., Sedykh, I.A, (2012), Discrete model in the class neighborhood systems, Bulletin of Tambov University. A series of Natural and technical Sciences. Tambov, T. 17. Vol, 3, pp. 867-871.

[9] Sedykh, I.A, (2016), Parametric identification of linear dynamic models neighborhoodCollection of articles of International scientific-practical conference "Innovation science: the past, present, future". Ufa: AETERNA, pp. 12-19.

[10] Shmyrin, A.M., Sedykh, I.A., Semina, V.V, (2016), Identification neighborhood neural network model based on a greedy, "poluianov" algorithms and algorithm Kachmazh, Vestnik TSU. Ser. Natural and technical Sciences, T. 21. Vol. 6, pp. 2121-2127.

[11] Shmyrin, A.M., Sedykh, I.A., Shcherbakov, A.P, (2014), A General bilinear discrete models, Herald of the Voronezh state technical University, T. 10, Vol. 3-1, pp. $44-49$.
[12] Shmyrin, A.M., Sedykh, I.A, (2008), Identification of linear systems neighborhood representing Petri nets, prospects of development of information technologies. The collection of materials of I all-Russian scientific-practical conference. Novosibirsk, pp. 91-97.

[13] Zainullin, R.R., Galyautdinov, A.A, (2016), Problems of urban wastewater, Innovative science, Vol. 6-2, pp. 68-69. 